\title{
ENDS OF LOCALLY COMPACT GROUPS AND THEIR COSET SPACES
}

\author{
Dedicated to the memory of Hanna Neumann
}

C. H. HOUGHTON

(Received 20 June 1972)

Communicated by M. F. Newman

\section{Introduction}

Freudenthal $[5,7]$ defined a compactification of a rim-compact space, that is, a space having a base of open sets with compact boundary. The additional points are called ends and Freudenthal showed that a connected locally compact noncompact group having a countable base has one or two ends. Later, Freudenthal [8], Zippin [16], and Iwasawa [11] showed that a connected locally compact group has two ends if and only if it is the direct product of a compact group and the reals.

Using Freudenthal's theory, Hopf [10] introduced a parallel theory of ends of finitely generated infinite discrete groups. Hopf showed that the number of ends is one, two, or is infinite and he characterised groups with two ends as being infinite cyclic by finite. Freudenthal [6] expressed the theory in combinatorial terms and this approach was used by Stallings [14] to characterise finitely generated groups with infinitely many ends. By formulating the theory algebraically, Specker [13] extended it to groups which are not finitely generated and showed that such groups have one or infinitely many ends.

Specker [13] also developed a theory of ends of locally compact Hausdorff groups, which generalises the theory for discrete groups and also the topological theory, when the group is sufficiently nice. We consider a generalisation of Specker's theory to right coset spaces $X=G / H$ where $G$ is locally compact Hausdorff and $H$ is a closed subgroup. A $Q$-end of $X$ is defined as an ultrafilter of non-compact closed subsets $S$ of $X$ such that $S V \backslash S$ is relatively compact for all relatively compact neighbourhoods $V$ of 1 in $G$. In the discrete case, the $Q$-ends of $G$ are the group ends of $G$. For connected $G$, we show that the $Q$-ends of $X=G / H$ coincide with the Freudenthal ends of $X$. Our proof depends on a result of Abels [1] that a connected locally compact group has a compact connected neighbourhood of the identity. We show that, for any $X$, the $Q$-ends of $X$ are the additional points in a zero-dimensional compactification $X^{*}$ of $X$ belonging to a general class discussed by Freudenthal [7]. 
Most of our results on the number of $Q$-ends are proved by analogy with corresponding results for discrete groups (see Cohen [3]). In Theorem 3.7, we characterise those $X=G / H$ having two $Q$-ends, under the assumption that $N / H$ is non-compact, where $N$ is the normaliser of $H$ in $G$. In Section 4 we show that if $G$ is compactly generated and $N / H$ is non-compact then the number of $Q$-ends is one, two, or infinite. If the connected component of 1 in $N / H$ is not compact, then $X$ has one or two $Q$-ends. Finally, if $G$ is locally compact and has a closed compactly generated subgroup $D$ such that $D$ and $G / D$ are not compact, then $G$ has one $Q$-end if $D$ is a normal subgroup, and otherwise has one or infinitely many $Q$-ends.

I am grateful to the referee for his comments, in particular, for informing me of the related work of Abels [1]. I thank Dr. Abels for a preprint of his paper and for drawing my attention to the paper of Specker [13].

Throughout the paper, all spaces considered will be Hausdorff.

\section{Compactifications}

We begin by describing a class of compactifications of a rim-compact space $X$, introduced by Freudenthal [7]. Suppose $A$ is a base of open sets with compact boundary such that if $S, T \in A$ then $S \cup T, S \cap T$ and $X \backslash S \in A$. A family of sets in $A$ is a binding family if the intersection of the closures of the sets in any finite subfamily is non-empty. We define $X^{*}$ to be the set of maximal binding families and take as a base for the open sets of $X^{*}$ all sets $\psi(S)=\left\{x^{*} \in X^{*}: \bar{U} \subseteq S\right.$ for some $\left.U \in X^{*}\right\}$, where $S \in A$. Then $X^{*}$ is a compact Hausdorff space and the map $x \rightarrow \phi(x)=\{S \in A: x \in \bar{S}\}$ is a topological embedding of $X$ in $X^{*}$; the space $X^{*} \mid X$ is zero-dimensional. In the case where $A$ consists of all open sets with compact boundary, $X^{*}=F X$ is called the Freudenthal compactification of $X$.

We find it useful to give a different description of the compactification $X^{*}$ defined above. Let $C$ be the base for the closed sets of $X$ consisting of the complements of all sets in $A$.

Lemma 2.1. The compactification $X^{*}$ of $X$ associated with $A$ is homeomorphic to the set $Y$ of $C$-ultrafilters with topology given by taking as a base for the closed sets all sets $\theta(T)=\{e \in Y: T \in e\}$, where $T \in C$. Each $x \in X$ corresponds to the ultrafilter of sets in $C$ containing $X$. In the case $X^{*}=F X, C$ consists of all closed sets with compact boundary.

Proof. If $I(T), F(T)$ denote respectively the interior and boundary of a closed set $T$, then $T=I(T) \cup F(T)$ and for $T \in C, I(T) \in A$ and $F(T)$ is compact. For $x^{*} \in X^{*}$ let $\alpha\left(x^{*}\right)=\left\{T \in C: T \cap \bigcap_{i=1}^{n} \bar{U}_{i} \neq \varnothing\right.$ for all subfamilies $\left\{U_{1}, \cdots, U_{n}\right\}$ of $\left.x^{*}\right\}$. We show $\alpha\left(x^{*}\right) \in Y$. Suppose firstly that for some $T \in \alpha\left(x^{*}\right), V=I(T) \notin x^{*}$. By Lemma 2 of Fan and Gottesman [4], there exists $W \in x^{*}$ such that $\bar{V} \cap \bar{W}=\varnothing$. Then for all $U_{1}, \cdots, U_{n} \in x^{*}, \bar{V} \cap \bar{W} \cap \bigcap_{i=1}^{n} \bar{U}_{i}=\varnothing ;$ but $T \cap \bar{W} \cap \bigcap_{i=1}^{n} \bar{U}_{i} \neq \varnothing$ 
so $D \cap \bigcap_{i=1}^{n} \vec{U}_{i} \neq \varnothing$ where $D=F(T)$. Thus the family of all subsets of form $D \cap \bigcap_{i=1}^{n} \bar{U}_{i}$ of the compact space $D$ has the finite intersection property and so for some $x \in D, x \in \bar{U}$ for all $U \in x^{*}$. Since $x^{*}$ is a maximal binding family, $x^{*}=\phi(x)$ and $\alpha\left(x^{*}\right)$ is the $C$-ultrafilter consisting of all sets $T \in C$ which contain $x$. In the remaining case, if $T \in \alpha\left(x^{*}\right)$ then $I(T) \in x^{*}$. Thus if $T_{1}, T_{2} \in \alpha\left(x^{*}\right)$ then $T_{1} \cap T_{2} \supseteq \overline{I\left(T_{1}\right)} \cap \overline{I\left(T_{2}\right)}$ and $\overline{I\left(T_{1}\right)} \cap \overline{I\left(T_{2}\right)} \cap \bigcap_{i=1}^{n} \bar{U}_{i} \neq \varnothing$ for all $U_{1}, \cdots, U_{n} \in x^{*}$ so $T_{1} \cap T_{2} \in \alpha\left(x^{*}\right)$. Hence $\alpha\left(x^{*}\right)$ is clearly a $C$-filter and if $S \in C$ and $S \cap T \neq \varnothing$ for all $T \in \alpha\left(x^{*}\right)$ then, since $\bigcap_{i=1}^{n} \bar{U}_{i} \in \alpha\left(x^{*}\right)$ for all $U_{1}, \cdots, U_{n} \in x^{*}$, we have $S \in \alpha\left(x^{*}\right)$ and so $\alpha\left(x^{*}\right)$ is a $C$-ultrafilter. Conversely, given a $C$-ultrafilter $e$, let $f$ denote the family of open sets with closure in $e$. Clearly $f$ is a binding family. If $e$ contains a compact set then $\bigcap_{T \in e} T=\{x\}$ for some $x \in X$ and $f=\phi(x), e=\alpha(f)$. Otherwise if $T=\overline{I(T)} \cup F(T) \in e$ then $\overline{I(T)} \in e$. It follows that $f$ is maximal and clearly $e=\alpha(f)$. Thus we have a bijection between $X^{*}$ and the set $Y$ of $C$-ultrafilters. This becomes a homeomorphism when we take as an open base for $Y$ all sets $\alpha \psi(S)=\{e \in Y: T \subseteq S$ for some $T \in e\}$, where $S \in A$. The result now follows.

Similar results have been obtained by Banaschewski [2] and Njåstad [12] with $C$ replaced by the set of finite intersections of closures of, respectively, the regular open sets in $A$ and the sets in $A$. Their methods apply to the more general compactifications of Fan and Gottesman [4], whereas our method does not. Suppose $C$ is any base of closed sets of $X$ with compact boundary such that if $S, T \in C$ then $S \cup T, S \cap T$, and $\overline{X \backslash T} \in C$. Then the set $A$ of complements of sets in $C$ satisfies Freudenthal's conditions and so we have an associated compactification $Y$ as defined in Lemma 2.1. In fact one can show that $C$ is a Wallmann basis, in the sense of Banaschewski [2], and then $Y$ is the associated Wallmann-type compactification. It is then straightforward to check directly that $Y \backslash X$ is zerodimensional.

Let $G$ be a locally compact group and denote by $B=B(G)$ the set of all relatively compact neighbourhoods of 1 in $G$. Let $H$ be a closed subgroup of $G$ and let $X$ be the right coset space $G / H$. We define $Q=Q(X)$ to be the set of all closed $S \subseteq X$ such that $S V \backslash S$ is relatively compact for all $V \in B$. Then $Q$ contains all compact subsets of $X$ and all closed subsets with relatively compact complements.

THEOREM 2.2. The sets in $Q$ have compact boundary and form a base for

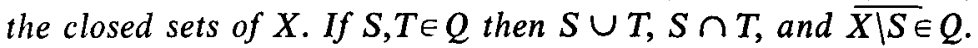

Proof. For $S \in Q$ and $V \in B,(X \mid S) V \cap S \subseteq\left(S V^{-1} \mid S\right) V$, which is relatively compact. Then

$$
F(S)=S \cap \overline{X \mid S}=S \cap \bigcap_{V \in B}(X \mid S) V=\bigcap_{V \in B}(X \mid S) V \cap S,
$$

so $F(S)$ is compact. Since $Q$ contains all closed sets with compact complements, 
it is a base for the closed sets of $X$. If $S, T \in Q$ and $V \in B$ then $(S \cup T) V=S V \cup T V$ and $(S \cap T) V \subseteq S V \cap T V$ so $(S \cup T) V \backslash(S \cup T) \subseteq(S V \backslash S) \cup(T V \backslash T)$ and $(S \cap T) V \backslash(S \cap T) \subseteq(S V \mid S) \cup(T V \backslash T)$. Finally, if $S \in Q$ and $\mathrm{V} \in B, \bar{X} \backslash \bar{S}$ $=(X \mid S) \cup F(S)$ so $(\overline{X \mid S}) V /(\overline{X \mid S}) \subseteq((X \mid S) V \cap S) \cup F(S) V$, which is relatively compact.

From now on, let $X^{*}$ denote the compactification of $X$ associated with $Q$. Thus $X^{*}$ is the space of $Q$-ultrafilters with a base for the closed sets given by all $\theta(T)=\left\{e \in X^{*}: T \in e\right\}$, where $T \in Q$. The embedding of $X$ is given by $x \rightarrow\{T \in Q: x \in T\}$, and $X^{*} \mid X$ is zero-dimensional. We call the elements of $X^{*} \mid X$ the $Q$-ends of $X$ and denote $X^{*} \mid X$ by $E(X)$. Since $X$ is locally compact, the $Q$-ends consist precisely of those $Q$-ultrafilters containing no compact sets. The elements of $F X \backslash X$ will be referred to as $F$-ends and, in the same way, they consist of those ultrafilters of closed sets with compact boundary which contain no compact sets.

If $G$ is discrete and $H$ is trivial then a subset $S$ of $G$ is in $Q$ if and only if $S V \backslash S$ is finite for all finite subsets $V$ of $G$ containing 1 . This is equivalent to the condition that $(S g \cap(G \backslash S)) \cup\left(S g^{-1} \cap(G \mid S)\right) g=(S g \cup S) \backslash(S g \cap S)$ is finite for all $g \in G$. Then $E(G)$ consists of all ultrafilters of infinite sets of this type, and this is precisely the usual definition of the set of ends of a discrete group (see Cohen [3]). At the other extreme, the next theorem shows that when $G$ is connected the $Q$-ends and $F$-ends of $G / H$ coincide. This generalises to coset spaces the result of Specker [13] for $H=1$. In our proof we use the result of Abels [1] that a connected locally compact group has a compact connected neighbourhood of 1 .

THEOREM 2.3. Let $G$ be a connected locally compact group with closed subgroup $H$. Then $X^{*}=F X$, where $X=G / H$.

Proof. It is sufficient to show that if $S \subseteq X$ is closed and has compact boundary then $S \in Q$. Let $W \in B$ and suppose $V$ is a symmetric compact connected neighbourhood of 1 . Then $V^{i}$ is connected for all $i$ and, since $G$ is connected, $G=\bigcup_{i=1}^{\infty} V^{i}$ and $W \subseteq V^{i}$ for some $i$. Then $S W \backslash S \subseteq S V^{i} \backslash S$ and the result will follow provided $S U \backslash S$ is relatively compact for any symmetric compact connected neighbourhood $U$ of 1 . But, for some $x_{1}, \cdots, x_{n} \in X, F(S) \subseteq \mathbb{U} \bigcup_{i=1}^{n} x_{i} U$. If $x \in S U \backslash S$ then $x U \cap S$ $\neq \varnothing \neq x U \cap(X \mid S)$ so $x U \cap F(S) \neq \varnothing$ and $x \in \bigcup_{i=1}^{n} x_{i} U^{2}$, which is relatively compact.

THEOREM 2.4. Let $K$ be a closed subgroup of $G$ containing $H$ and such that $K / H$ is compact. Then the natural map $\pi: G / H \rightarrow G / K=Y$ induces a continuous map from $E(X)$ onto $E(Y)$ which is a homeomorphism if $K$ is normal in $G$ and $H=1$.

Proof. We note that $\pi$ and $\pi^{-1}$ preserve relative compactness and that $\pi$ is a closed map. If $S \in Q(X)$ and $V \in B$ then $\pi(S) V \backslash \pi(S)=\pi(S V) \backslash \pi(S) \subseteq \pi(S V \backslash S)$ so $\pi(S) \in Q(Y)$. Conversely if $T \in Q(Y)$ then, for $V \in B$, 


$$
\pi^{-1}(T) V\left|\pi^{-1}(T)=\pi^{-1}(T V)\right| \pi^{-1}(T) \subseteq \pi^{-1}(T V \backslash T),
$$

which is relatively compact, so $\pi^{-1}(T) \in Q(X)$. If $e \in E(X)$ and $S, T \in e$, then $\pi(S) \cap \pi(T) \supseteq \pi(S \cap T) \in \pi(e)$ and it follows that $\pi(e)$ is a subbase for a $Q(Y)$ filter $\pi_{1}(e)$. If $T \in Q(Y)$ and $T \cap \pi(S) \neq \varnothing$ for all $S \in e$, then $\pi^{-1}(T) \cap S \neq \varnothing$ for all $S \in e$ and so $\pi^{-1}(T) \in e$. Then $T \in \pi(e)$ and hence $\pi_{1}(e)=\pi(e)$ is a $Q(Y)$ ultrafilter which clearly contains no compact sets. If $f \in E(Y)$ then $\pi^{-1}(f)$ is a subbase for a $Q(X)$-filter and $f$ is then the image under $\pi$ of those $Q(X)$-ultrafilters containing $\pi^{-1}(f)$. Thus $\pi: X \rightarrow Y$ extends to a map from $X^{*}$ onto $Y^{*}$ which is clearly continuous and takes $E(X)$ onto $E(Y)$.

Suppose $K$ is normal in $G$ and $H=1$; so $X=G$. For $f \in E(Y), \pi^{-1}(f)$ is a subbase for a $Q(G)$-filter. Suppose $S_{1}, S_{2} \in Q(G)$ and $S_{1} \cap \pi^{-1}(T) \neq \varnothing \neq S_{2} \cap \pi^{-1}(T)$ for all $T \in f$. Then $\pi\left(S_{1}\right) \cap T \neq \varnothing \neq \pi\left(S_{2}\right) \cap T$ so $\pi\left(S_{1}\right), \pi\left(S_{2}\right) \in f$ and hence $\pi\left(S_{1}\right) \cap \pi\left(S_{2}\right)$ is not compact. Then $S_{1} K \cap S_{2} K=\pi^{-1}\left(\pi\left(S_{1}\right)\right) \cap \pi^{-1}\left(\pi\left(S_{2}\right)\right)$ $=\pi^{-1}\left(\pi\left(S_{1}\right) \cap \pi\left(S_{2}\right)\right)$ is not compact. Since $K$ is compact, $K \subseteq V$ for some $V \in B$. Then $S_{1} K\left|S_{1} \subseteq S_{1} V\right| S_{1}$ and $S_{2} K\left|S_{2} \subseteq S_{2} V\right| S_{2}$ so $S_{1} K \mid S_{1}$ and $S_{2} K \mid S_{2}$ are relatively compact and $S_{1} \cap S_{2} \neq \varnothing$. Thus $\pi^{-1}(f)$ is contained in a unique $Q(G)$ ultrafilter and the restriction of $\pi$ to $E(G)$ is injective. Let $\sigma: E(Y) \rightarrow E(G)$ denote the inverse of the restriction of $\pi$ to $E(G)$. A base for the closed sets of $E(G)$ is given by all $\mu(S)=\{e \in E(G): S \in e\}$, where $S \in Q(G)$, and then $\pi(\mu(S))$ $\subseteq\{f \in E(Y): \pi(S) \in f\}$. Given $f \in E(Y)$ such that $\pi(S) \in f$, then $\pi^{-1}(\pi(S))=S K \in \sigma(f)$. Now $K \subseteq V$ for some $V \in B$, and then $S K \backslash S \subseteq S V \backslash S$, which is relatively compact. Hence $S \in \sigma(f)$ and $f \in \pi(\mu(S))$. Thus $\pi(\mu(S))=\{f \in E(Y): \pi(S) \in f\}$, which is closed in $E(Y)$. Hence $\sigma$ is continuous and so $E(G)$ and $E(Y)$ are homeomorphic.

The assumption that $K$ is normal is essential for the last part. For let $G$ be the discrete infinite dihedral group $\left\langle a, b: b^{2}=b^{-1} a b a=1\right\rangle$ and let $K=\langle b\rangle$. Then (see Cohen [3] or Theorem 3.7 below) $G$ has $2 Q$-ends but it is straightforward to check that any set in $Q(G / K)$ is finite or has finite complement and so $G / K$ has just $1 Q$-end.

THEOREM 2.5. Let $L$ be a closed subgroup of $G$ containing $H$ such that $G / L$ is compact. Then $E(X)$ and $E(L / H)$ are homeomorphic.

Proof. Let $Z=L / H$. Since $G / L$ is compact, there is a compact subset $C$ of $G$ such that $G=L C$. If $V \in B(G)$ is closed and symmetric, then $W=V \cup C \cup C^{-1}$ is a closed symmetric member of $B(G)$ and $G=L W, X=Z W$. If $U \in B(G)$, then $U=U \cap L W \subseteq(U W \cap L) W$ and clearly $U W \cap L \in B(L)$. Thus if $T \in Q(Z)$ and $V \in B(G), T W V \backslash T W \subseteq T(W V W \cap L) W \backslash T W \subseteq(T(W V W \cap L) \backslash T) W$, which is relatively compact, so $T W \in Q(X)$.

If $S \in Q(X)$ and $V \in B(L)$ then $V \subseteq V W \in B(G)$ and $(S \cap Z) V /(S \cap Z)$ $\subseteq Z \cap(S V \mid S)$ so $S \cap Z \in Q(Z)$. Now $S=S \cap Z W \subseteq(S W \cap Z) W \subseteq((S W \backslash S)$ $\cup(S \cap Z)) W$ so, if $S \cap Z$ is compact, $S$ is also compact. Thus if $e \in E(X)$, 
$\{S \cap Z: S \in e\}$ is a subbase for a $Q(Z)$-filter. Suppose $T_{1}, T_{2} \in Q(Z)$ and $T_{1} \cap S \cap Z$ $\neq \varnothing \neq T_{2} \cap S \cap Z$ for all $S \in e$. Then $T_{1} W \cap S \neq \varnothing \neq T_{2} W \cap S$ and so $T_{1} W$, $T_{2} W \in e$ and $T_{1} W \cap T_{2} W$ is not compact. Now $T_{1} W \cap T_{2} W \subseteq\left(T_{1} \cap T_{2} W^{2}\right) W$ and since the cosets in $T_{1}$ and $T_{2}$ are contained in $L$, we have

$$
T_{1} \cap T_{2} W^{2}=T_{1} \cap T_{2}\left(W^{2} \cap L\right)=\left(T_{1} \cap T_{2}\right) \cup\left(T_{1} \cap\left(T_{2}\left(W^{2} \cap L\right) \backslash T_{2}\right)\right) .
$$

Thus $\left(T_{1} \cap T_{2}\right) W$ is not compact and $T_{1} \cap T_{2} \neq \varnothing$. Hence $\{S \cap Z: S \in e\}$ is contained in a unique $Q(Z)$-ultrafilter $\tau(e)$. If $T \in \tau(e)$ then $T W \in e$ so $\tau(e)$ contains no compact sets and $\tau(e) \in E(Z)$.

For $f \in E(Z),\{T W: T \in f\}$ is a subbase for a $Q(X)$-filter. Suppose $S_{1}, S_{2} \in Q(X)$ and $S_{1} \cap T W \neq \varnothing \neq S_{2} \cap T W$ for all $T \in f$. Then $S_{1} W \cap T \neq \varnothing \neq S_{2} W \cap T$, so $S_{1} W \cap Z, S_{2} W \cap Z \in f$ and thus $S_{1} W \cap S_{2} W \cap Z$ is not compact. Now $S_{1} W \cap S_{2} W$ $\subseteq\left(S_{1} W^{2} \cap S_{2}\right) W \subseteq\left(\left(S_{1} W^{2} \mid S_{1}\right) \cup\left(S_{1} \cap S_{2}\right)\right) W$, so $S_{1} \cap S_{2} \neq \varnothing$. Thus $\{T W: T \in f\}$ lies in a unique $Q(X)$-ultrafilter $\sigma(f)$. If $S \in \sigma(f)$ then $S W \cap Z \in f$ so $\sigma(f)$ contains no compact sets and $\sigma(f) \in E(X)$. If $T \in f$ then $(T W \cap Z) \backslash T \subseteq(T \cap Z W)(W \cap L) \backslash T$ $=T(W \cap L) / T$, which is relatively compact, so $T W \cap Z \in f$ and hence $\tau \sigma(f)=f$. Similarly, if $e \in E(X)$ and $S \in e$ then $S W \in e$ and, since $(S W \cap Z) W \backslash S \subseteq S W^{2} \mid S$ is relatively compact, $(S W \cap Z) W \in e$ and hence $\sigma \tau(e)=e$. Given $S \in Q(X)$ and $e \in E(X), S \in e$ if and only if $S W \cap Z \in \tau(e)$, so $\tau$ is a ciosed map. Given $T \in Q(Z)$ and $f \in E(Z), T \in f$ if and only if $T W \in \sigma(f)$ and so $\sigma$ is a closed map. Thus $E(X)$ and $E(Z)$ are homeomorphic.

\section{Coset spaces with two ends}

In this section we assume that $G$ is compactly generated and hence there is a compact symmetric neighbourhood $V$ of 1 such that $G=\bigcup_{i=1}^{\infty} V^{i}$. Then if $W \in B, W \subseteq V^{i}$ for some $i$. Also $X=G / H=\left\{H g: g \in \bigcup_{i=1}^{\infty} V^{i}\right\}$ where, as before, $H$ is a closed subgroup of $G$.

LemMa 3.1. In this situation, a closed subset $S$ of $X$ is in $Q$ if and only if $S V \mid S$ is relatively compact.

PROOF. For $i, j \geqq 1, S V^{i+j} \mid S \subseteq\left(S V^{i} \mid S\right) V^{j} \cup\left(S V^{j} \mid S\right)$, so if $S V \backslash S$ is relatively compact, $S V^{i} \mid S$ is also relatively compact, for $i \geqq 1$. If $W \in B$ then $W \subseteq V^{i}$ for some $i$ and $S W \backslash S \subseteq S V^{i} \mid S$. The result now follows.

If $W$ is a symmetric neighbourhood of 1 in $G$, we say $S \subseteq X$ is $W$-connected if, given any two elements $x, y \in S$, there is a $W$-chain in $S$ joining them, that is, there exist $w_{1}, \cdots, w_{n} \in W$ such that $y=x w_{1} \cdots w_{n}$ and $x w_{1} \cdots w_{m} \in S$ for $m \leqq n$.

Lemma 3.2. If $V \in B$ is symmetric and $G=\bigcup_{i=1}^{\infty} V^{i}$ then each $S \in Q$ is $V^{j}$. connected for some $j$.

Proof. If $R=(X \mid S) V \cap S$ then $R \subseteq(S V \mid S) V$, so $R$ is relatively compact. 
Now $X$ is $V$-connected so we may assume $S \neq X$. Let $T$ be a maximal $V$-connected subset of $S$. Then there is a $V$-chain from a point in $T$ to a point in $X \backslash S$ and, by the maximality of $T$, for some $y \in T, v \in V$ we have $y v \in X \mid S$. Then $y \in R$ so $T \cap R$ $\neq \varnothing$. Let $U \in B$ be symmetric and such that $U^{2} \subseteq V$. Since $R$ is relatively compact, $R \subseteq \bigcup_{i=1}^{n} x_{i} U$ for some $x_{i} \in X$. If $T_{1}, T_{2}$ are maximal $V$-connected subsets of $S$ and for some $x_{i}, T_{1} \cap x_{i} U \neq \varnothing \neq T_{2} \cap x_{i} U$ then $T_{1} V \cap T_{2} \neq \varnothing$. Thus the number of maximal $V$-connected subsets of $S$ is finite. If $H y_{1}, \cdots, H y_{m}$ are representatives of the different ones, with $y_{i} \in G$, then for some $j, y_{1}^{-1} y_{i} \in V^{j}$ for $i=1, \cdots, m$, and so $S$ is $V^{j}$-connected.

LEMMA 3.3. Let $G$ be compactly generated and let $N$ be the normaliser of $H$ in $G$. Suppose $S \in Q$. For $T \in Q$, let $L \subseteq N$ consist of those $g \in N$ such that $g T \subseteq S$ or $X \mid g T \subseteq S$. If $\pi: G \rightarrow G \backslash H=X$ denotes the natural map, then $S \cap \pi(N \backslash L)$ is relatively compact.

Proof. Let $U=\overline{X \mid S}$. Then $U \in Q$ and, by Lemma 3.2 , we may assume $U$ is $V$-connected, where $V \in B$ is symmetric and $G=\bigcup_{i=1}^{\infty} V^{i}$. If $g \in M=N \backslash L$, then $g T \cap U \neq \varnothing \neq(X \mid g T) \cap U$ and, since $U$ is $V$-connected, there are elements $y \in g T \cap U, v \in V$ such that $y v \in(X \mid g T) \cap U$. Thus if $R=T V / T, g R \cap U \neq \varnothing$. Now $R$ is relatively compact so $R \subseteq \pi\left(V^{i}\right)$ for some $i$. Thus for $g \in M, \varnothing \neq g \pi\left(V^{i}\right)$ $\cap U=\pi\left(g V^{i}\right) \cap U$ and $\pi(g) \subseteq U V^{i}$. Thus $\pi(M) \subseteq U V^{i}$ and, since $S=(X \mid U)$ $\cup(S \cap U), S \cap \pi(M) \subseteq\left(U V^{i} \mid U\right) \cup(S \cap U)$, which is relatively compact.

LEMMA 3.4. Let $G$ be compactly generated and let $\pi: G \rightarrow G / H=X$ be the natural map. Suppose $S \in Q$ is such that $S$ and $U=\overline{X \mid S}$ are not compact and let $K \subseteq N=N_{G}(H)$ consist of those $g \in N$ for which $(g S \cap U) \cup(g U \cap S)$ is compact. If $\pi(K)$ is not relatively compact then $G=H C W$, where $C$ is a discrete infinite cyclic subgroup of $N$ with $H \cap C=1$ and $W$ is a compact symmetric neighbourhood of 1 . Then $|E(X)|=2$.

Proof. Changing the roles of $S$ and $U$ if necessary, we may assume $\pi(K) \cap U$ is not relatively compact. Since $F(S)$ is compact, $I(S) \neq \varnothing$. Take $x \in S$ and an open symmetric $V \in B$ such that $x V \subseteq S$. Then $T=S \cap(X \mid x V) \in Q$. By Lemma 3.3, with $U$ replacing $S$, there is an element $c^{-1} \in K$ such that $c^{-1} T \subseteq U$ or $X \mid c^{-1} T \subseteq U$. But $c^{-1} T \cap U \subseteq c^{-1} S \cap U$, which is compact, so $c^{-1} T \supseteq X \mid U$ and $c^{-1} T \supseteq \overline{X \mid U}=S$. Then $c S \subset S$ and $c^{n} S \subset S$ for all $n>0$, so $c^{n} \notin H$ and, if $C=\langle c\rangle, C$ is infinite with $C \cap H=1$ and $C \leqq N$. Suppose $R$ is a relatively compact subset of $X$ and $c^{n} x \in R$ for infinitely many $n$. For some $x_{1}, \cdots, x_{r} \in X$, $R \subseteq x_{1} V \cup \cdots \cup x_{r} V$ and hence for some $m, n, i$ with $m>n, c^{m} x, c^{n} x \in x_{i} V$. Then $c^{m} x V \cap c^{n} x V \neq \varnothing$ and $c^{m-n} x V \cap x V \neq \varnothing$. But $c^{m-n} x V \subseteq c^{m-n} S \subseteq c S \subseteq T$ $\subseteq X \mid x V$. This contradiction shows that any relatively compact subset of $X$ contains only finitely many $c^{n} x$. Hence $C$ is not relatively compact and so is a discrete subgroup of $G$. 
For $n>1, \quad c^{n} S \subseteq X \mid x V$ so $c^{n} S \cap x V=\varnothing$ and $c^{-n} x \in X \mid S$. Suppose $z \in \bigcap_{n=1}^{\infty} c^{n} S$. Then $z=x g^{-1}$ for some $g \in G$ and $c^{-n} x \in S g$ for $n>1$. Now $\{1, g\} \subseteq V_{0}$, for some $V_{0} \in B$, and, for $n>1, c^{-n} x \in S g \cap(X \mid S) \subseteq S V_{0} \mid S$, which is relatively compact. This contradicts a previous remark and we conclude that $\bigcap_{n=1}^{\infty} c^{n} S=\varnothing$. If $n>0, c^{n} x V \subseteq S$ and, since $S \cap U=F(S)$ is compact, for some $k, c^{n} x \in X \backslash U$ for $n \geqq k$. An argument similar to the previous one now shows $\bigcap_{n=k}^{\infty} c^{-n} U=\varnothing$. For $n \geqq 0, c^{n} S \subseteq S$ so $X \mid S \subseteq c^{n}(X \mid S)$ and $c^{-n} U \subseteq U$. Thus

$$
\begin{aligned}
X & =S \cup U=\bigcup_{n=0}^{\infty}\left(S \backslash c^{n} S\right) \cup \bigcup_{n=0}^{\infty}\left(U \backslash c^{-n} U\right) \\
& =\bigcup_{n=0}^{\infty}\left(c^{n} S \backslash c^{n+1} S\right) \cup \bigcup_{n=0}^{\infty}\left(c^{-n} U \backslash c^{-n-1} U\right) \\
& \subseteq C((S \backslash c S) \cup(c U \backslash U)) \subseteq C(S \cap c U) .
\end{aligned}
$$

Since $c \in K, S \cap c U$ is compact and so for some compact symmetric neighbourhood $W$ of 1 in $G, S \cap c U=\pi(W)$ and hence $G=H C W$. Since $C$ is discrete, it is a closed subgroup of $G$ and so $H C$ is closed in $G$. Now $H C / H$ is a discrete infinite cyclic group and so has $2 Q$-ends and, since $G / H C$ is compact, $G / H$ has $2 Q$-ends, by Theorem 2.5 .

The results of Freudenthal [8], Zippin [16], and Iwasawa [11], characterise a connected locally compact group with two $F$-ends as being the direct product of a compact group and the reals. Hofmann and Mostert [9] consider the class of locally compact groups which can be compactified by adding two non-isolated points and they characterise those for which the connected component of 1 has compact coset space. Groups with two $Q$-ends are in this class, but so are all discrete groups. We use the following result.

THEOREM 3.5. (Hofmann and Mostert $[9$, p. 63, 64]). Let $G$ be a locally compact group such that the component of the identity is not compact. Suppose $G$ has a discrete infinite cyclic subgroup with compact coset space. Then either $G$ or a subgroup of index two in $G$ is a direct product of the reals and a normal compact subgroup $L$ of $G$. In the second case $G / L$ is topologically isomorphic to the split extension of the reals by the involution $x \rightarrow-x$.

To complete the description of groups with two $Q$-ends we use the characterisation of discrete groups with two ends and the following weak form of a result of $\mathrm{Wu}$.

THEOREM 3.6. ( $W u[15])$. Let $G$ be a locally compact totally disconnected group containing a discrete free abelian subgroup having compact coset space. Then $G$ has normal subgroups $K, L$ with $K \geqq L$ such that $G / K$ and $L$ are compact and $K / L$ is discrete. 
THEOREM 3.7. Let $H$ be a closed subgroup of a compactly generated group G. Suppose $N / H$ is not compact, where $N=N_{G}(H)$, and let $N_{0} / H$ be the connected component of 1 in $N / H$. Then $|E(G / H)|=2$ if and only if $N / H$ has normal subgroups $M / H$ and $L / H$, with $M \geqq L$, such that $G / M$ and $L / H$ are compact and (i) if $N_{0} / H$ is compact, $M / L$ is discrete infinite cyclic or infinite dihedral, (ii) if $N_{0} / H$ is not compact, either $M / H$ or a subgroup of index 2 in $M / H$ is the dirct product of $L / H$ and a group topologically isomorphic to the reals; in the latter case, $M / L$ is isomorphic to the split extension of the reals by the involution $x \rightarrow-x$.

Proof. If $G / H$ has the described form then, by Theorems 2.4 and 2.5 , $|E(G / H)|=|E(M / L)|$. Now $M / L$ has a discrete infinite cyclic subgroup having compact coset space and so $|E(G / H)|=2$. Conversely, if $X=G / H$ has $2 Q$-ends $e$ and $f$ then for some $S \in Q, S \in e$ and $U=\bar{X} \mid \bar{S} \in f$, so $S$ and $U$ are not compact. The left action of $N$ on $X^{*}$ clearly permutes $e$ and $f$ and, if $K$ consists of the elements of $N$ leaving the ends fixed, $|N / K|=1$ or 2 . If $g \in K$ and $g S \cap U$ is not compact, then $g S \cap U \in f$ and $S \cap g^{-1} U \in f$. Then $f$ contains the compact set $S \cap g^{-1} U \cap U$, which is impossible. Thus for $g \in K,(g S \cap U) \cup(g U \cap S)$ is compact. If $\pi: G \rightarrow X$ is the natural map then $\pi(K)$ is not compact so, by Lemma 3.4, $G=H C W$ with $C$ a discrete infinite cyclic subgroup of $N$ and $W$ compact. Then $G / N$ is compact and the group $\pi(N)$ has a discrete infinite cyclic subgroup with compact coset space. In case (ii), the result, with $M=N$, is now immediate from Theorem 3.5 since $\pi(N)$ has $2 Q$-ends.

In case (i), $\pi\left(N_{0}\right)$ is compact and $N_{1}=\pi(N) / \pi\left(N_{0}\right)$ is a totally disconnected locally compact group having a discrete infinite cyclic subgroup with compact coset space. By Theorem 3.6, $N_{1}$ has normal subgroups $N_{2}, N_{3}$, with $N_{2} \geqq N_{3}$, such that $N_{1} / N_{2}$ and $N_{3}$ are compact and $N_{2} / N_{3}$ is discrete. By Theorems 2.4 and 2.5, $\left|E\left(N_{2} / H_{3}\right)\right|=|E(X)|=2$ and so (see Cohen [3]) $N_{2} / N_{3}$ has a finite normal subgroup $N_{4} / N_{3}$ such that $N_{2} / N_{4}$ is infinite cyclic or dihedral. Taking $M$ and $L$ as the inverse images of $N_{2}$ and $N_{4}$ respectively under the natural map $\pi(N) \rightarrow N_{1}$, we obtain the result.

\section{The number of ends}

THEOREM 4.1. Let $G$ be compactly generated with closed subgroup $H$ and let $X=G / H, N=N_{G}(H)$. If $N / H$ is not compact, then $|E(X)|=1,2$, or is infinite.

Proof. Suppose $E(X)$ is finite. Then the left action of $N$ on $E(X)$ induces a finite group of permutations of $E(X)$ so $N$ has a normal subroup $M$, with $N / M$ finite, such that $M$ leaves the $Q$-ends fixed. Let $S \in Q$ be non-compact and put $U=\overline{X / S}$. Then, as in the proof of Theorem 3.7, for $m \in M,(m S \cap U) \cup(m U \cap S)$ is compact. Now $M / H$ is not compact so, by Lemma 3.4, if $U$ is not compact, 

$|E(X)|=2$. Otherwise, $\overline{X \mid S}$ is compact for all non-compact $S \in Q$ and so
$E(X) \mid=1$.

THEOREM 4.2. Let $G$ be compactly generated with closed subgroup H. Suppose that the connected component of 1 in $N / H$ is not compact. Then $|E(X)|=1$ or 2 .

Proof. Let $\pi: G \rightarrow G / H$ be the natural map; we note that $\pi$ restricted to $N$ is a homomorphism. Abels [1] shows that a connected locally compact group has a compact connected neighbourhood of 1 . Thus we may choose a symmetric neighbourhood $W$ of $H$ in $N$ such that $\pi(W)$ is compact and connected. If $L=\bigcup_{1=0}^{\infty} W^{i}$, then $\pi(L)=\bigcup_{i=0}^{\infty} \pi\left(W^{i}\right)=\bigcup_{i=0}^{\infty} \pi(W)^{i}$, which is the component of 1 in $N / H$. Let $g \in L$. Then $g \in W^{i}$, for some $i$, and if $S \in Q$ and $x \in g S \cap(X \mid S)$ then $W^{i} x \cap S \neq \varnothing \neq W^{i} x \cap(X \mid S)$. Now if $x=\pi(k), W^{i} x=\pi\left(W^{i} k\right)=\pi\left(W^{i}\right) k$ $=\pi(W)^{i} k$ which is connected since $\pi(W)$ is connected. Then $W^{i} x \cap F(S) \neq \varnothing$ and $x \in W^{i} F(S)$. Thus if $U=\overline{X \mid S}, g S \cap U \subseteq(g S \cap(X \mid S)) \cup F(S) \subseteq W^{i} F(S)$. Since $F(S)$ is compact, $F(S)=\pi(V)$ for some compact $V \subseteq G$ and $W^{i} F(S)$ $=\pi\left(W^{i} V\right)=\pi(W)^{i} V$, which is compact. Thus for all $g \in L, g S \cap U$ is compact and similarly $g U \cap S$ is compact. Since $\pi(L)$ is not compact, Lemma 3.4 implies $|E(X)|=1$ or 2 .

So far we have assumed $G$ is compactly generated. The final result holds for more general locally compact groups.

THEOREM 4.3. Let $G$ be a locally compact group having a closed compactly generated subgroup $D$ such that $D$ and $G / D$ are not compact. Then $|E(G)|=1$ or is infinite. If $D$ is a normal subgroup then $|E(G)|=1$.

Proof. Suppose $E(G)$ is finite. As in the proof of Theorem 4.1, $G$ has a normal subgroup $M$ of finite index whose left action fixes the $Q$-ends of $G$. By Theorem 2.5, $|E(G)|=|E(M D)|$ and from the proof, $M$ fixes the $Q$-ends of $K=M D$.

Suppose $S$ is in $Q(K)$ and is not compact and let $U=\bar{K} \mid \bar{S}$. Then $(m S \cap U)$ $\cup(m U \cap S)$ is compact for all $m \in M$. Let $D=\bigcup_{i=0}^{\infty} V^{i}$ where $V \in B$. Then $R=(S V \mid S) \cup(U V \backslash U)$ is relatively compact. For $k \in K, k D$ is $V$-connected so either $k D \subseteq S, k D \subseteq U$ or $k D \cap R \neq \varnothing$ and $k \in R D$. Since $G / D$ is not compact, neither is $K / D$ and we may assume $g D \subseteq S$ for some $g \in M$. If $h \in M$ then $h D \cap U=h g^{-1}\left(g D \cap g h^{-1} U\right) \subseteq h g^{-1}\left(S \cap g h^{-1} U\right)$, which is compact. Thus for $k \in K$, either $k D \subseteq S$ or $k \in R D$ and $k D \cap U$ is compact. Since $R$ is relatively compact, $R \subseteq \bigcup_{i=1}^{n} x_{i} V$ for some $x_{i} \in R$ and so $U=K \cap U=R D \cap U$ $\subseteq \bigcup_{i=1}^{n}\left(x_{i} D \cap U\right)$, which is compact. Thus $|E(G)|=1$.

We now consider the case where $D$ is normal and impose no restriction on $|E(G)|$. Suppose $S \in Q(G)$ is not compact and let $U=\overline{G \mid S}$. If $R=(S V \mid S) \cup(U V \mid U)$ then, as above, for $g \in G$, either $g D \subseteq S, g D \subseteq U$ or $g \in R D$. Since $G / D$ is not compact, we may assume $g D \subseteq S$ for some $g \in G$. If $h \in G$, then $h D \cap U=D h \cap U$ 
$=\left(D g \cap U h^{-1} g\right) g^{-1} h \subseteq\left(S \cap U h^{-1} g\right) g^{-1} h$, which. $s$ compact. As above, it follows that $U$ is compact and hence $|E(G)|=1$.

For $X=G / H$, the argument we have used where $D$ is not necessarily normal can only be extended to the case in which $G / N_{G}(H)$ is compact. But, in that case, it follows anyway fron the theorem and Lemma 2.5 that $|E(X)|=1$ or is infinite. In the case where $D$ is normal, the argument extends to show that $|E(X)|=1$ if we have $D \leqq N_{G}(H)$ and neither $D H / H$ nor $G / D$ is compact.

Finally, we note that if $G$ is a locally compact group having a closed compactly generated non-compact subgroup, then either $G$ satisfies the hypotheses of Theorem 4.3 or it is compactly generated. Thus $|E(G)|=1,2$ or is infinite, the case $|E(G)|=2$ being determined in Theorem 3.7.

\section{References}

[1] H. Abels, 'Enden von Räumen mit eigentlichen Transformationsgruppen', Comment. Math. Helv. 47 (1972), 457-473.

[2] B. Banaschewski, 'On Wallmann's method of compactification', Math. Nach. 27 (1963), 105-114.

[3] D. E. Cohen, Groups of cohomological dimension one, (Springer-Verlag, Berlin, 1972).

[4] Ky Fan and N. Gottesman, 'On compactifications of Freudenthal and Wallmann', Indag. Math. 14 (1952), 504-510.

[5] H. Freudenthal, 'Neuaufbau der Endentheorie', Ann. of Math. 43 (1942), 261-279.

[6] H. Freudenthal, 'Über die Enden diskreter Räume and Gruppen', Comment. Math. Helv. 17 (1944), 1-38.

[7] H. Freudenthal, 'Kompaktisierungen und Bikompaktisierungen', Indag. Math. 13 (1951), 184-192.

[8] H. Freudenthal, 'La structure des groupes à deux bouts et des groupes triplement transitifs', Indag. Math. 13 (1951), 288-294.

[9] K. H. Hofmann and P. Mostert, 'Splitting in topological groups', Mem. Amer. Math. Soc. 43 (1963).

[10] H. Hopf, 'Enden offener Räume und unendliche diskontinuerliche Gruppen', Comment. Math. Helv. 16 (1943), 81-100.

[11] K. Iwasawa, 'Topological groups with invariant neighbourhoods of the identity', Ann. of Math. 54 (1951), 345-348.

[12] O. Njåstad, 'On Wallmann-type compactifications', Math. Z. 91 (1966), 267-276.

[13] E. Specker, 'Endenverbände von Räumen und Gruppen', Math. Ann. 122 (1950), 167-174.

[14] J. Stallings, Groups theory and three-dimensional manifolds, (Yale Mathematical Monographs 4, 1971).

[15] T. S. Wu, 'A certain type of locally compact totally disconnected topological group', Proc. Amer. Math. Soc. 23 (1969), 613-614.

[16] L. Zippin, 'Two-ended topological groups', Proc. Amer. Math. Soc. 1 (1950), 309-315.

Department of Pure Mathematics

University College, Cardiff

Wales 